\title{
What phonological facilitation tells about semantic interference: a dual-task study
}

\section{Pauline Ayora ${ }^{*}$, Francesca Peressotti ${ }^{2}$, F.-Xavier Alario ${ }^{1,3}$, Claudio Mulatti ${ }^{2}$, Patrick Pluchino ${ }^{2}$, Remo Job ${ }^{4}$ and Roberto Dell'Acqua ${ }^{2,5}$}

1 Laboratoire de Psychologie Cognitive, Aix-Marseille Université and CNRS, Marseille, France

2 Department of Developmental Psychology, University of Padova, Padova, Italy

${ }^{3}$ Center for Mind/Brain Sciences, University of Trento, Rovereto, Italy

${ }^{4}$ Department of Cognitive Science, University of Trento, Rovereto, Italy

${ }^{5}$ Center for Cognitive and Brain Science, University of Padova, Padova, Italy

\section{Edited by:}

Eduardo Navarrete, University of

Padova, Italy

\section{Reviewed by:}

Victor Ferreira, University of California

San Diego, USA

Kevin Dent, University of Birmingham UK

${ }^{*}$ Correspondence:

Pauline Ayora, Laboratoire de Psychologie Cognitive, Aix-Marseille Université and CNRS, Centre St Charles, 3 Place Victor Hugo (Bâtiment 9, Case D), 13331 Marseille, France. e-mail: pauline.ayora@univ-provence.fr
Despite increasing interest in the topic, the extent to which linguistic processing demands attentional resources remains poorly understood. We report an empirical re-examination of claims about lexical processing made on the basis of the picture-word interference task when merged in a dual-task psychological refractory period (PRP) paradigm. Two experiments were conducted in which participants were presented with a tone followed, at varying stimulus onset asynchronies (SOAs), by a picture-word stimulus. In Experiment 1, the phonological relatedness between pictures and words was manipulated. Begin- and end-related words decreased picture naming latencies relative to unrelated words. This effect was additive with SOA effects. In Experiment 2, both the semantic and the phonological relatedness between pictures and words were manipulated. Replicating Experiment 1, effects arising from the phonological manipulation were additive with SOA effects on picture naming latencies. In contrast, effects arising from the semantic manipulation were under additive with SOA effects on picture naming latencies, that is, semantic interference decreased as SOA was decreased. Such contrastive pattern suggests that semantic and phonological effects on picture naming latencies are characterized by distinguishable sources, the former prior to the PRP bottleneck and the latter at the PRP bottleneck or after. The present findings are discussed in relation to current models of language production.

Keywords: language production, dual-task, picture-word interference, phonological facilitation, semantic interference

\section{INTRODUCTION}

Producing even the simplest utterance belies an articulate sequence of processing stages and representations. Once a speaker has encoded a conceptual message to be expressed, she has to access to the linguistic representations that will be used to convey it. In particular, she has to select words that best correspond to the intended message and retrieve the appropriate phonological codes that will ultimately drive the verbal message articulation (for overview, see Levelt et al., 1999). A central topic in this domain of studies concerns the stage at which words are selected prior to articulation. The picture-word interference (PWI) paradigm has been used extensively as a potentially diagnostic means to locate the lexical selection stage in the word production architecture. For example, one typical effect obtained in PWI paradigms is the longer naming latency observed when a to-be-named picture is displayed concurrently with a to-be-ignored word that is semantically related (HORSE-cat) relative to when they are unrelated (HOUSE-cat, e.g., Schriefers et al., 1990). A number of researchers have associated this so-called semantic interference effect to lexical, post-lexical, and pre-lexical stages of processing. The lexical selection hypothesis attributes the semantic interference effect to the central lexicalization stage, considering that activation levels of semantically related non-target words slow down the time it takes to select the target word (Schriefers et al., 1990; Roelofs, 1992; Levelt et al., 1999). The post-lexical hypothesis locates the semantic interference effect at a response exclusion stage (Mahon et al., 2007), where response is triggered for articulation. According to this view, distractor words have privileged access to the articulatory output buffer, and the time it takes to remove the distractor from this buffer is function of its semantic relevance for the naming task at hand. A third class of interpretation has highlighted the role of pre-lexical processes involved in message elaboration at the semantic level (e.g., Costa et al., 2005; Kuipers et al., 2006). According to this view, the conflict created by the semantic interplay between pictures and words occurs at the level of conceptual processes of message elaboration, to select which semantic representation will be lexicalized.

Current attempts at establishing the relative order of processing stages along the chain underpinning word production have turned to employ the psychological refractory period (PRP) paradigm. In the PRP paradigm, two stimuli (S1 and S2) are presented at varying stimulus onset asynchronies (SOAs). Each stimulus is associated with a distinct reaction time (RT1 and RT2) task. One or both tasks (Task1 and Task2) engage central attention mechanisms, which are critical for various mental operations, such as response selection (Pashler and Johnston, 1998), mental rotation (Van Selst and Jolicoeur, 1994), short-term memory consolidation (Jolicoeur and 
Dell'Acqua, 1998), or grammatical processing (Ayora et al., 2009). A crucial assumption underlying the PRP paradigm is that such central mechanisms operate serially on sequential stimuli. Under conditions of task overlap (i.e., at short SOA), central processing stages in Task 2 are temporarily suspended until analogous processing stages in Task 1 are finished. In contrast, pre-central and postcentral mechanisms engaged for one task occur in parallel with any other stages of the other task (see Pashler, 1994, for a review). When both tasks require central mechanisms, manipulations prolonging pre-central or central stages of Task1 processing prolong RT2 by a corresponding amount. In contrast, manipulations prolonging post-central stages of Task1 processing do not have any influence on RT2, since processing in both tasks take place in parallel (e.g., Pashler and Johnston, 1989; Dell'Acqua and Jolicoeur, 2000).

Ferreira and Pashler (2002) applied this logic in a language production study. They tested the classic semantic interference effect in Task1 while participants had to discriminate the pitch of a tone in Task2. The semantic interference effect found in Task1 propagated to Task2. In line with the logic outlined above, this finding ruled out a post-central locus of the semantic interference effect, leaving open the possibility of a pre-central or central locus of this effect. The importance of the locus of the semantic interference effect in current thinking about lexical processing motivated a complementary study by Dell'Acqua et al. (2007a) where task order was reversed. In this situation, manipulations prolonging pre-central stages of processing in Task2 (picture naming; see Figure 1) should only be manifest at long SOA, since at short SOA RT2 effects are hypothesized to be absorbed into the period of Task 2 suspension. In contrast, manipulations prolonging central or post-central stages of processing in Task2 should produce additive effects with SOA. Dell'Acqua et al. (2007a) showed that semantic interference effects detected in Task2 were fully manifest at long SOA, and virtually absent at short SOA (i.e., an under additive interaction). This ruled out the potential of a central locus of the PWI semantic effect, and suggested a pre-central locus of the semantic interplay between pictures and words in PWI tasks.

Dell'Acqua's et al. (2007a) finding, however, is prone to alternative interpretations. Cohen and Magen (2004) observed under additive effects of visual congruency with SOA in a flanker task. They interpreted this pattern as evidence for focused visual encoding at short SOAs, favoring target information over distracting information. Lien et al. (2008) invoked Cohen's and Magen (2004) findings to argue that, at short SOAs, visual attention may either actively inhibit distractor word processing, or allow privileged processing of pictures at the expense of words in a PWI paradigm. In either case, such inefficient distractor word processing hypothesis could provide an alternative account of the under additive semantic interference pattern of Dell'Acqua et al. (2007a). In other words, the under additivity of SOA and semantic interference in PWI would not be symptomatic of the locus of the semantic interplay between pictures and words, but of the different attention allocation policy affecting targets and distractors processing across SOAs.

One hypothetical case that could represent a serious challenge for the inefficient distractor word processing would be the finding of additive effects of SOA and of a manipulation of PWI picture/ word relatedness in the context of a PRP paradigm. As is presently formulated, the inefficient distractor word processing hypothesis is tacit about the nature of the relationship between a to-be-named picture and a potentially distracting word displayed in the PWI task. The tenet is simply that, in a combination of PWI and PRP paradigms, visual words at short SOA are disregarded more efficiently when the task-relevant information is represented by the picture.

To test the generality of the inefficient distractor word processing hypothesis, two PWI/PRP experiments were devised in the present investigation in which both the semantic relatedness and phonological relatedness were manipulated in the PWI task implemented as Task 2 in a PRP design. The well-known phonological facilitation effect is characterized by shorter picture naming latencies when both stimuli are phonologically related (CUP-cat) than when they are unrelated (PEN-cat, e.g., Meyer and Schriefers, 1991). In previous PRP studies, the phonological facilitation effect has only been implemented in Task 1 and led to contrasting results. Ferreira and Pashler (2002) did not observe any phonological facilitation propagation to RT2. By contrast, Cook and Meyer (2008) observed such propagation when using masked distractors. Roelofs (2008) observed phonological facilitation propagation when the auditory S2 was replaced by a visual stimulus. More recently, Mulatti

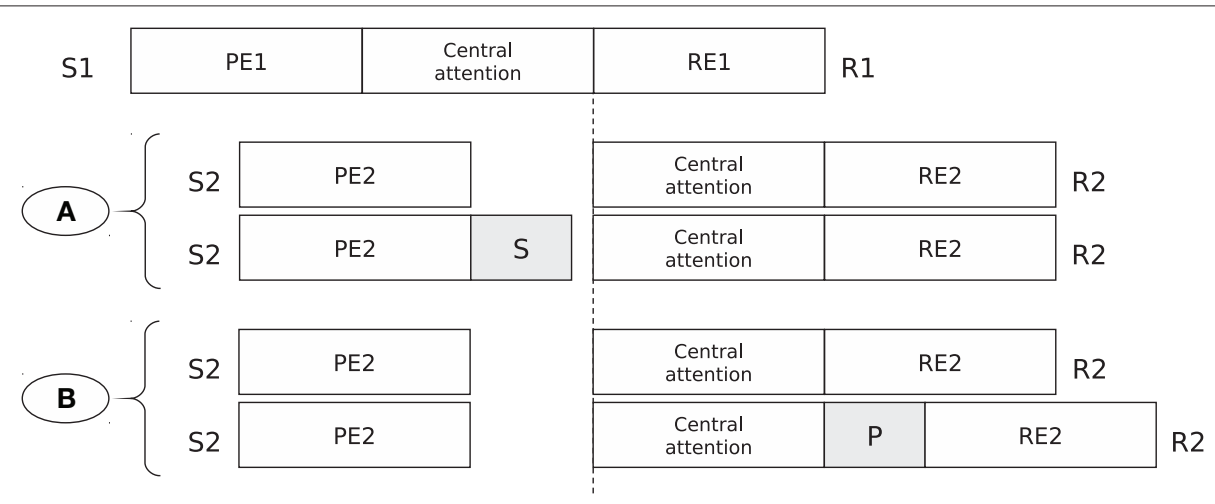

FIGURE 1 | (A) Gant diagrams illustrating the account of the under additivity between SOA and semantic effects (shaded box labeled S) proposed by Dell'Acqua et al. (2007a). (B) Gant diagrams illustrating the account of the additivity between SOA and phonological effects (shaded box labeled P) and the under additivity between SOA and semantic effects (shaded box labeled S) proposed in the present study. In the boxes, PE, perceptual encoding; RE, response execution. Numbers in the boxes are mapped to task order (1:Task1, S1 = tone, R1 = manual; 2: Task2, $\mathrm{S} 2$ = picture and word superimposed, R2 = vocal response) 
et al. (submitted) provided evidence nicely dovetailing with that obtained by Ferreira and Pashler (2002), showing in addition that when the distractor does not belong to the response set (i.e., the distractor words are not names of other pictures in the experiment), the phonological facilitation effect does not propagate to RT2. The discussion of these findings has been centered on the central vs. post-central locus of the phonological effects. Irrespective of the conclusions reached in that debate, however, both loci predict that phonological effects should produce additive effects when inserted in Task2 (see Discussion above, and Figure 1B), providing that the distractor words are indeed processed at short as well as long SOAs. To reiterate, under an inefficient distractor word processing hypothesis, Task 2 suspension at short SOA would allow the visual processing system to focus on the pictures at the expense of the distractor words. Thus, the phonological facilitation should produce under additive effects with SOA on RT2. If such under additive pattern were observed, we would not be able to decide between the inappropriateness of the PRP paradigm to investigate PWI effects, or a pre-central stage of processing for phonological (and semantic) effects. In contrast, observing additive effects between phonological relatedness and SOA would indicate that the printed distractor word is processed even at the shortest SOA. This would provide a much safer ground for considering that semantic distractors are indeed processed at short SOAs, and possibly concluding that semantic interference is pre-central while phonological facilitation is central or post-central.

\section{EXPERIMENT 1 MATERIALS AND METHODS \\ Participants}

Twenty-eight students of the University of Padova volunteered to participate in the present experiment. All reported having learned Italian as their first language and having no vision or hearing disturbance.

\section{Apparatus and stimuli}

The set of 48 pictures used by Dell'Acqua et al. (2007a) was used in the present experiment. Each picture was paired with two related distractor words selected from the CoLFIS corpus (Laudanna et al., 1995), one sharing the initial phonemes (begin-related) and one sharing the final phonemes (end-related) with picture names. For each phonologically related distractor word a phonologically unrelated word was selected. Related and unrelated words were matched for length and lexical frequency (all $t s<1$ ). The font (Romantri 32 ) and mean length of the words $(t<1)$ did not differ from those used by Dell'Acqua et al. (2007a). The words were displayed at the center of the monitor surrounded by the pictures, all within a square portion of the monitor measuring $6^{\circ} \times 6^{\circ}$, controlled by MEL software. The same apparatus was used to generate the acoustic stimuli (pure tones of frequencies 300,600, $1200 \mathrm{~Hz}$ ) and control vocal/manual response recordings.

\section{Design and procedure}

Begin- and end-related lists were randomly assigned to two distinct groups of participants. Within a list, the 288 trials (i.e., 48 pictures $\times 2$ distractors $\times 3$ SOAs) were presented in a different randomized order to each participant, and organized in 12 blocks of 24 trials each. In each block, the different combinations of SOA and picture-word phonological relatedness were randomly intermixed and equiprobable. The experimental stimuli were preceded by a block of 24 practice trials with stimuli that were not included in the experimental list.

Each trial began with the presentation of a fixation point for $1000 \mathrm{~ms}$, followed by a blank interval of $800 \mathrm{~ms}$, and by the presentation of a randomly selected tone (S1) for $50 \mathrm{~ms}$. At an SOA of 100,350 , or $1000 \mathrm{~ms}$, a picture-word compound stimulus (S2) was displayed. Participants were instructed to discriminate the tone pitch by pressing their index, medium, and annular fingers on three arrayed keys of a response box, and to name the picture as fast and accurately as possible while ignoring the word. The instructions stressed the importance to respond first to the tone stimulus.

\section{RESULTS}

Correct reaction times shorter than $100 \mathrm{~ms}$ and longer than $4500 \mathrm{~ms}$ ( 0.89 and $1.0 \%$ of RT1s and RT2s, respectively) as well as trials in which the response to Task 1 was given after the response to Task2 $(0.010 \%)$ were discarded from analysis. Error rates and average latencies associated with trials with a correct response in both tasks (RT1 and RT2) were submitted to separate analyses of variance (ANOVAs) considering subject and items as random factors $(F 1, F 2)$. SOA and phonological relatedness were analyzed within participants and items; position of shared phonemes (initial vs. final) was analyzed between participants and within items.

\section{Accuracy}

Error rates in Task1 were affected by SOA $[F 1(2,52)=11.3$, $\eta p^{2}=0.303, p<0.001 ;$ F2 $\left.(2,94)=5.5, \eta p^{2}=0.104, p<0.01\right]$. From the shortest to the longest SOAs, the proportion of errors was $0.12,0.10$, and 0.10 , respectively. A separate ANOVA in which the data from the shortest SOA were temporarily excluded from consideration showed that SOA effects were no longer significant $(F 1$ and $F 2<1)$. No significant effects were detected in the analysis of error rates in Task2 (all Fs $<=1$ ).

\section{Reaction time 1}

A summary of the results is illustrated in Figure 2. The only significant effect on RT1 was that of SOA $\left[F 1(2,52)=22.4, \eta p^{2}=0.462\right.$, $\left.p<0.001 ; F 2(2,94)=10.1, \eta p^{2}=0.177, p<0.001\right]$, reflecting a modest increase of RT1 at the shortest SOA relative to the two longer SOAs. Positive correlations between RT1 and RT2 at short SOAs may have a variety of sources, such as response grouping (i.e., the tendency to wait to emit a Task1 response until a Task2 response is also ready to be emitted), graded resource sharing between tasks, or momentary distraction from Task1 due to S2 abrupt onset. In the present study, RT1 increase from the shortest to the longest SOAs was $18 \mathrm{~ms}$, and this, per se, suggests that effects due to any combination of the above factors were modest. More importantly, PRP studies have reported that SOA-dependent modulations of RT1 have no modulatory influence on the interaction (additive or under additive) between SOA and Task 2 variables as reflected on RT2 (e.g., Ruthruff et al., 2001). A separate ANOVA in which the data from the shortest SOA condition were temporarily excluded from analysis showed that SOA effects on RT1 were no longer significant $(F 1$ and $F 2<1)$. 


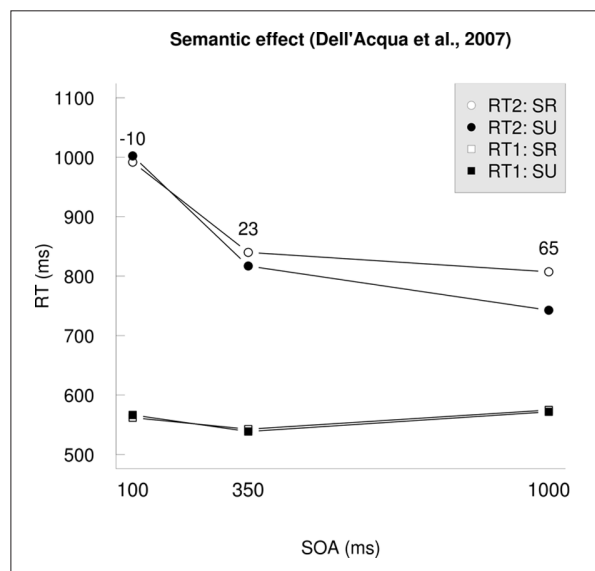

FIGURE 2 | Left panel: Results of the semantically related manipulation reported in Dell'Acqua et al. (2007a). Center Panel: Results for the beginrelated phonological manipulation in Experiment 1. Mean reaction times, plotted separately for tone discrimination (RT1) and picture naming latencies (RT2), as a function of phonological relatedness, and SOA. PR = picture and word
Phonological effect (Initial phonemes)

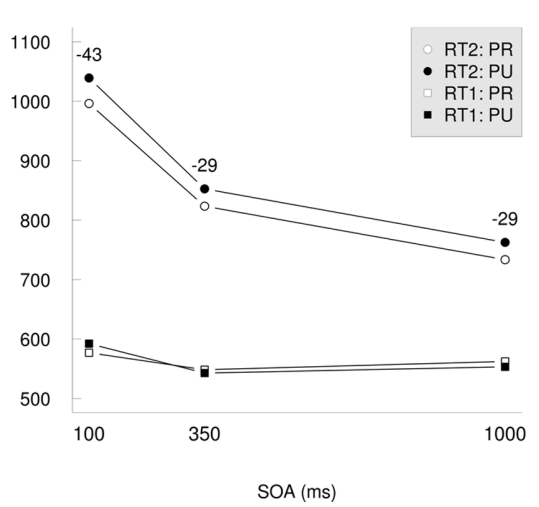

Phonological effect (Final phonemes)

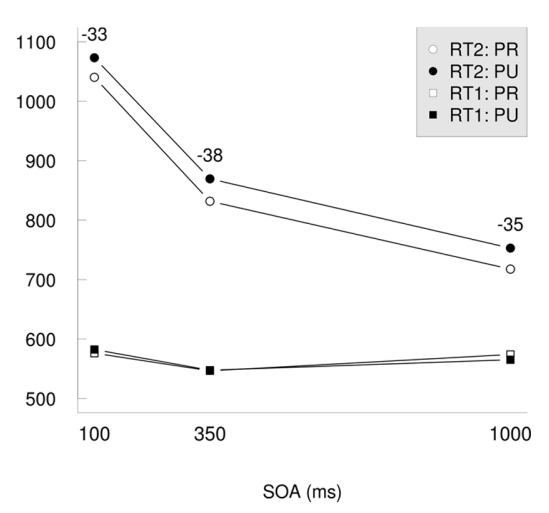

phonologically related; PU = picture and word phonologically unrelated. Right panel: Results for the end-related phonological manipulation in Experiment 1. Numerical values indicate the magnitudes of the semantic interference effect obtained in Dell'Acqua et al. (2007a; left panel) and of the phonological facilitation effects obtained in Experiment 1 (center and right panels).

\section{Reaction time 2}

Reaction time 2 increased as SOA decreased $[F 1(2,52)=288.5$, $\eta p^{2}=0.897, p<0.001 ;$ F2 $\left.(2,94)=584.32, \eta p^{2}=0.921, p<0.001\right]$. RT2 was shorter with phonologically related distractors than with unrelated distractors $\left[F 1(1,26)=47.2, \eta p^{2}=0.646, p<0.001 ; F 2(1\right.$, $\left.47)=18.5, \eta p^{2}=0.283, p<0.001\right]$. These two factors did not interact $(F 1$ and $F 2<1)$. The overall magnitude of the phonological effects at the shortest, intermediate, and longest SOAs was 38, 34, and $32 \mathrm{ms,}$ respectively. The interaction between $\mathrm{SOA}$, phonological relatedness and relatedness position was not significant (both $F s<1$ ), indicating that the additive pattern of SOA and relatedness did not depend on the position (initial vs. final) of the critical phonemes (Figure 2). A main effect of related phoneme position was detected only in the by-item analysis $\left[F 2(1,47)=10.0, \eta p^{2}=0.176, p<0.01\right]$, reflecting that distractor words sharing their initial phonemes with the picture names induced more facilitation than distractor words sharing their final phonemes with the picture names. The interaction between related phoneme position and SOA was significant in the by-item analysis only $\left[F 2(2,94)=11.0, \eta p^{2}=0.19, p<0.001\right]$. This interaction reflects that the previous main effect of related phoneme position extended only to the two shortest SOAs. It is not clear why this interaction arose. The important point to note here is that the critical phonemes position factor did not interact with any of the other factors considered in the RT2 analyses (both F1s and F2s $<1$ ).

\section{Cross-comparison between experiments}

The results from the current experiment were compared to those of Dell'Acqua's et al. (2007a) in an omnibus by-subject and by-item ANOVA (F1 and F2). The analysis of RT2 revealed a significant three-way interaction between experimental condition, SOA, and picture-word relationship $\left[F 1(4,78)=4.3, \eta p^{2}=0.181, p<0.001\right.$; $\left.F 2(4,188)=2.5, \eta p^{2}=0.049, p<0.05\right]$. This significant interaction provides critical statistical support to the difference in the patterns reported graphically in the three panels of Figure 2. Whereas semantic and SOA effects were under additive in Dell'Acqua et al. (2007a), phonological and SOA effects were additive in the present study.

\section{DISCUSSION}

Experiment 1 showed that the phonological manipulation in the PWI Task2 and SOA produced additive effects. This implies that phonological distractor words were processed with equal efficiency across all the tested SOAs. As surmised in the Introduction, the present results are at odds with predictions derived from the inefficient distractor word processing assumption, and more compatible with the view that phonological effects are likely to have a central or post-central source, whereas semantic effects are likely to have a pre-central source in PWI tasks. Three distinct arguments related to the depth of processing distractors in PWI/PRP tasks must be considered carefully before drawing any firm conclusions from Experiment 1.

First, it may be argued that the additive pattern reported here with phonological manipulations reflects surface letter-to-phoneme mapping processes, and not lexical processing. To test this hypothesis, we conducted post hoc analyses on a subset of items that varied in lexical frequency of the distractor word, moving from the assumption that evidence of a distractor frequency effect (Miozzo and Caramazza, 2003) on RT2 would corroborate further the idea that distractor words were processed beyond the sub-lexical level (e.g., at the lexical level). Due to counterbalancing constraints, such analysis was conducted only on the begin-related condition and on Dell'Acqua's et al. (2007a) data. The word frequency measures available are expressed in occurrences per three millions (CoLFIS corpus; Laudanna et al., 1995). From the current materials, we selected 23 high frequency distractors (Min. $F=50$; Mean $F=88.91$, Mean length $=6.87$ letters) and 23 low frequency distractors (Max. $F=20$; Mean $F=10.57$, Mean length $=6.65$ letters). The pictures associated with these distractors were matched for name frequency and age of acquisition (Mean F: 64 and 54; Mean AoA: 3.03 and 3.15 ; $t<<1$ ). Eleven of the high frequency words and 12 of the low frequency words were phonologically (begin-) related to the picture name. From Dell'Acqua's et al. (2007a) experiment, we selected 22 high frequency distractors (Min. $F=40$; Mean $F=90$; Mean length $=6.2$ letters) and 22 low frequency distractors $($ Max. $F=12$; 
Mean $F=6$; Mean length $=7.07$ letters). The pictures associated with the high and low frequency distractor words were matched for name frequency and age of acquisition (Mean F: 50 and 51; Mean AoA: 2.9 and 3.08; ts $<1$ ). Eleven of the high frequency words and 11 of the low frequency words were also semantically related to the picture name.

For begin-related distractors, the ANOVA showed additive main effects of SOA $\left[F 1(2,26)=54.8, \eta p^{2}=0.79, p<0.001, F 2(2\right.$, $\left.88)=171.0, \eta p^{2}=0.77, p<0.001\right]$ and frequency $[F 1(1,13)=8.1$, $\left.\eta p^{2}=0.371, p<0.02, F 2(1,44)=4.4, \eta p^{2}=0.10, p<0.05\right]$ and no interaction $(F 1<1, F 2=1.6)$. Similarly, in the data from Dell'Acqua et al. (2007a), there was a significant effect of SOA $[F 1(2,26)=74.9$, $\left.\eta p^{2}=0.811, p<0.001, F 2(2,84)=101.8, \eta p^{2}=0.703, p<0.001\right]$, an effect of frequency significant by participants and marginally significant by-items $\left[F 1(1,13)=29.1, \eta p^{2}=0.69, p<0.02\right.$, $\left.F 2(1,42)=3.4, \eta p^{2}=0.069, p<0.08\right]$ and no interaction $(F 1<1$, $F 2=1.5$ ). Additive effects of distractor frequency and SOA (see also Mulatti et al., submitted, for similar findings) make it hard to hypothesize that distractor processing was confined to sub-lexical levels in Experiment 1.

Second, even assuming that distractors were processed lexically, one may still argue that what was bottlenecked at central stages at short SOA was the activation of the corresponding nodes at the semantic level of representation, leading to the under additive pattern observed by Dell'Acqua et al. (2007a). On the assumption that part of this dynamics could be strategic (e.g., Meyer and Kieras, 1997), one would expect subjects be able to exert some form of control on the magnitude of semantic interference in PWI tasks when strongly encouraged to ignore the words. This is contradicted by the study of Reiner and Morrison (1983). Early and automatic activation of semantic representations in the PWI task have, on the other hand, been recently described in an ERP study by Dell'Acqua et al. (2010). The semantic interference effect in the ERP timelocked to the onset of a picture-word stimulus was characterized bimodally by one component with a peak at about $120 \mathrm{~ms}$ and a second component with a later peak at $320 \mathrm{~ms}$, a latency comparable to the one obtained for phonological distractor words (see also Dell'Acqua et al., 2007b). Thus, it seems reasonable to consider that semantic activation from distractor words occurs rapidly and is unimpeded in picture naming under PWI conditions.

Third, and most importantly, it must be contemplated that whereas semantically related distractors tend to hamper naming latencies, phonologically related distractors produce facilitatory effects. After a variable number of trials in which subjects inadvertently paid attention to the nature of the words displayed concomitantly with the pictures, one option that cannot be excluded based on the results of Experiment 1 is that subjects adopted the strategy to rely on the information conveyed by distractor words so as to maximize their performance efficiency. This concern motivated Experiment 2, in which the same participants were exposed to picture-word stimuli that were unpredictably semantically related, phonologically related, or unrelated. If the previous results were due to different collateral strategy effects across experiments, then we should find a similar pattern of results for phonological and semantic distractor words. That is, semantic interference effect should appear at short SOA (along with the phonological facilitation effect) or the phonological facilitation effect should disappear at short
SOA (along with the semantic interference effect). In contrast, if the semantic interference effect were still absent at short SOA, and the phonological effect still present, this would corroborate our idea of different structural and functional origins of semantic interference and phonological facilitation in PWI tasks.

\section{EXPERIMENT 2 MATERIALS AND METHODS Participants}

Eighteen students of the University of Padova volunteered for the experiment. None took part to the previous experiment and all reported having learned Italian as their first language and having no vision or hearing disturbance.

\section{Apparatus and stimuli}

A subset of 35 pictures were selected from the 48 pictures used in Experiment 1 (mean name frequency $=42$; mean name length $=6.77$ letters). Each picture was paired with a semantically related distractor word, a phonologically related distractor word and an unrelated word, selected from the CoLFIS corpus (Laudanna et al., 1995), and only partially corresponding to the words used in Experiment 1 and in Dell'Acqua et al. (2007a; see Appendix for details). The phonologically related distractors always shared the first two or three phonemes with the target picture (i.e., begin-related). The semantically related distractors referred to exemplars belonging to the same semantic category as the target picture. Unrelated distractors never shared phonological segments, nor were related in meaning with the target word. Semantically related, phonologically related, and unrelated distractors were matched for frequency and length $(t s<1)$. The words were displayed in the center of the computer screen (resolution $640 \times 480$ ) in Courier New 20 font surrounded by the pictures, which occupied a portion of the screen subtending $7.8^{\circ} \times 7.8^{\circ}$ of visual angle. The stimuli were displayed in black on a white background. As in Experiment 1, the acoustic stimuli were pure tones of 300,600 , and $1200 \mathrm{~Hz}$. Stimulus presentation and response recording were controlled by a computer running E-Prime software (Schneider et al., 2002).

\section{Design and procedure}

The 210 stimuli (i.e., 35 pictures $\times 3$ distractors $\times 2$ SOAs) were presented in a different randomized order to each participant, and organized in six blocks of 35 trials each. At the end of each block, a feedback consisting in the mean RT in that block for task was presented visually. At the beginning of the session, each participant was first presented with the set of pictures to be named during the experiment with the corresponding names and, then, with the three tones that participants heard through headphones while the corresponding label "high $=1$," "medium $=2$," or "low $=3$ " appeared on the screen for five times in randomized order. The response to the tone was given using the keys 1,2, or 3 of the numeric keypad of the keyboard connected to the CPU controlling the experiment. The experimental phase was preceded by a block of 21 practice trials with stimuli that were not included in the experimental list. In this phase, a feedback on response accuracy to the tone and on the correct sequencing of the two responses (i.e., first tone discrimination then picture naming) was given at the end of each trial. The trial 
consisted of the same event sequence as in Experiment 1, with the exception of the SOAs tested in Experiment 2 that were reduced to two, 100 and $1000 \mathrm{~ms}$.

\section{RESULTS}

Correct reaction times shorter than $100 \mathrm{~ms}$ and longer than $4500 \mathrm{~ms}$ ( 0.91 and $1.12 \%$ of RT1s and RT2s, respectively) and trials in which the response to Task1 was given after the response to Task2 $(0.027 \%)$ were discarded from analysis. Error rates and average latencies associated with trials with a correct response in both tasks (RT1 and RT2) were submitted to separate ANOVAs considering subject and items as random factors (F1, F2). SOA (100 vs. $1000 \mathrm{~ms}$ ) and distractor type (phonologically related, semantically related, and unrelated) were analyzed within participants and items.

\section{Accuracy}

Error rates in Task1 were affected by SOA $\left[F 1(1,17)=24.1, \eta p^{2}=0.59\right.$, $\left.p<0.001 ; F 2(1,34)=92.1, \eta p^{2}=0.73, p<0.001\right]$. The proportion was 0.165 and 0.081 , at the short and long SOA, respectively. The effect of SOA was also significant in the analysis of error rates in Task2 [F1(1, 17) $\left.=4.9, \eta p^{2}=0.22, p=0.04 ; F 2(1,34)=10.4, \eta p^{2}=0.23, p=0.003\right]$, with more naming errors at the long $(0.10)$ than at the short $(0.07)$ SOA. This pattern of results is uncommon in the PRP literature and hard to interpret in the present design. However, given the absence of a significant interaction with the type of distractor factor (all $F_{\mathrm{S}}<1$ ), in no way is this pattern conceptually intertwined with the conclusion we draw from Experiment 2 concerning the locus of semantic interference and phonological facilitation in the present PWI/PRP design.

\section{Reaction time 1}

A summary of the results is illustrated in Figure 3. The ANOVA on RT1 did not reveal any significant effects (all Fs $<=1$ ).

\section{Reaction time 2}

Reaction time 2 increased as SOA decreased $[F 1(1,17)=131.3$, $\eta p^{2}=0.89, p<0.001 ;$ F2 $\left.(1,34)=2983.5, \eta p^{2}=0.99, p<0.001\right]$. A main effect of distractor type was also detected in the analysis $\left[F 1(2,34)=15.2, \eta p^{2}=0.47, p<0.001 ; F 2(2,68)=10.2, \eta p^{2}=0.23\right.$, $p<0.001]$. The interaction between SOA and distractor type was not significant $(F=1.7 ; p>0.2)$. Two ANOVAs were conducted separately on the data from the phonological condition and from the semantic condition (each contrasted separately with the unrelated condition). The ANOVA on the phonological data indicated significant effects of SOA $\left[F 1(1,17)=133.4, \eta p^{2}=0.88, p<0.001 ; F 2(1\right.$, $\left.34)=1064.6, \eta p^{2}=0.96, p<0.001\right]$, distractor type $[F 1(1,17)=17.0$, $\left.\eta p^{2}=0.50, p<0.001 ; F 2(1,34)=11.0, \eta p^{2}=0.24, p=0.002\right]$, and no interaction between the two factors $(F<1)$. The ANOVA on the semantic data indicated significant effects of SOA $[F 1(1,17)=109.5$, $\left.\eta p^{2}=0.87, p<0.001 ; F 2(1,34)=1291.3, \eta p^{2}=0.97, p<0.001\right]$, and of the interaction between SOA and distractor type $[F 1(1,17)=4.6$, $\left.\eta p^{2}=0.21, p=0.04 ; F 2(1,34)=3.4, \eta p^{2}=0.09, p=0.076\right]$.

\section{DISCUSSION}

The results replicate both Experiment 1 and Dell'Acqua et al. (2007a) within the same experiment, with partially new materials and with a different software controlling the experiment. Once

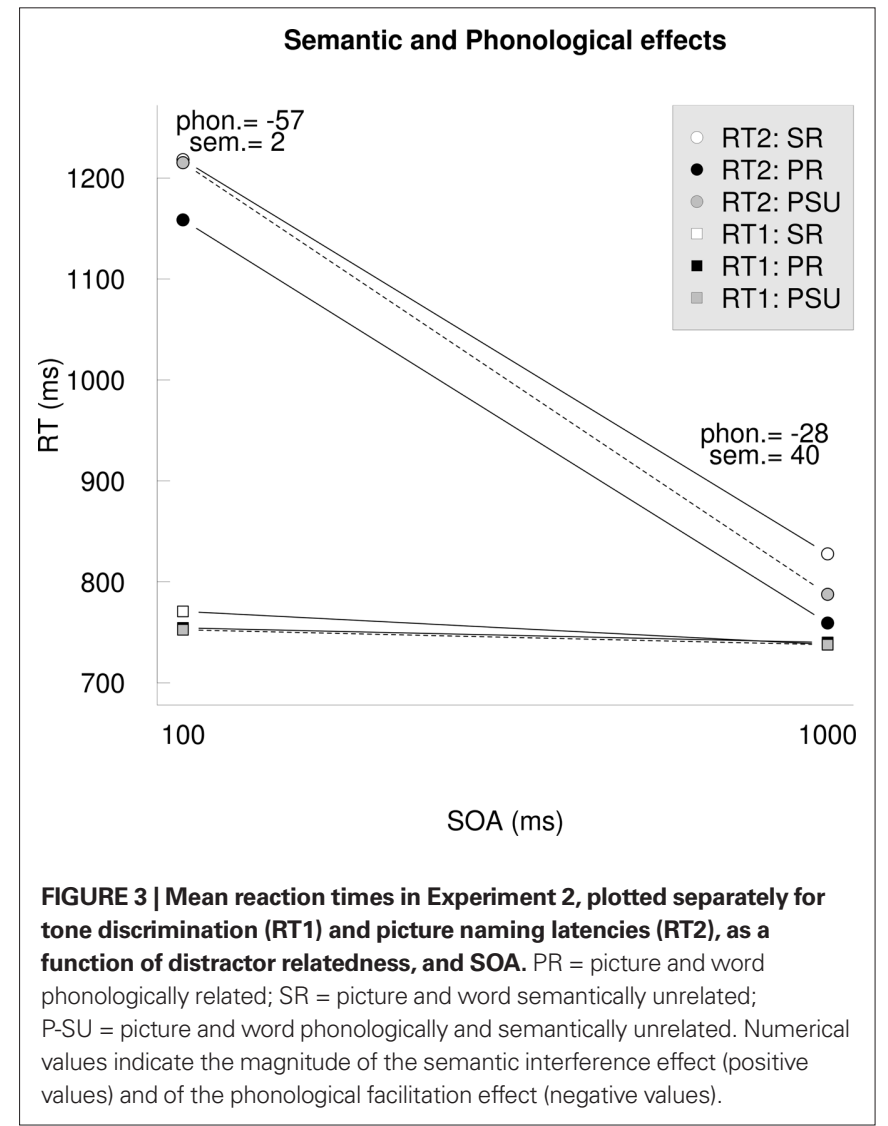

again, the results show additive effects between SOA and phonological facilitation and under additive effects between SOA and semantic interference. This finding helps us to rule out the suspect that the different patterns associated to phonological and semantic effects across experiments could be due to different strategies used by participants when exposed to distractor words sharing different relationship (e.g., phonological vs. semantic) with the to-be-named pictures in PWI tasks.

\section{GENERAL DISCUSSION}

In the present study, we investigated phonological and semantic effects on picture naming in a PWI task implemented as Task2 of a PRP design. At stake was whether, in this mixed PWI/PRP paradigm, inefficient distractor processing could account for the under additive pattern of semantic relatedness and SOA first documented by Dell'Acqua et al. (2007a) and replicated herein in Experiment 2. In Experiment 1, phonological effects were generated via a manipulation implemented in Task 2 that minimized the possible interference from ongoing speech encoding mechanisms on tone discrimination (e.g., Roelofs, 2008). The manipulation we used falls into the "canonical" PWI task (vis-à-vis variants in which, for instance, masking was adopted to minimize the impact of speech monitoring; e.g., Cook and Meyer, 2008). Furthermore, the fact that both begin- and end-related distractor words produced the same pattern of additivity with SOA clearly indicates that distractor words were processed as a whole entity under the present circumstances. As an important integration, we found 
evidence of additivity of SOA and distractor frequency effects both in Experiment 1 and in Dell'Acqua et al. (2007a) experiment, suggesting strongly that distractor words gained access to lexical representational format unconstrained by the PRP limitation. Thus, the additive effects of phonological relatedness and SOA provided the necessary support for claiming that distractor words can be processed sufficiently deeply and with equal efficiency at the different SOAs tested in the present PWI/PRP paradigm. This set of results runs against the inefficient distractor word processing hypothesis raised to provide an account of the under additive effects of semantic relatedness and SOA different from that put forth originally by Dell'Acqua et al. (2007a).

In Experiment 2, we manipulated phonological and semantic relatedness within participants, so as to minimize the possibility on the part of subjects to adopt a strategy bound to the type of distractor words, whether phonologically or semantically related. The results, that is, additivity of SOA and phonological facilitation and under additivity of SOA and semantic interference, are in line with the hypothesis of a different origin of phonological and semantic interplay between pictures and words in PWI tasks. Based on the so-called locus-of-slack logic, we argue that the most parsimonious explanation of the present dissociation is one that localizes the source of semantic interference effects at pre-central stages of processing during speech production and the source of phonological facilitation effects at central or post-central stages of processing.

An alternative explanation that we cannot exclude at present emerges from the observation that semantic and phonological effects persist when pictures and words are temporally staggered (i.e., when words and pictures are displayed asynchronously, at $\pm 100 \mathrm{~ms}$ from each other) in canonical PWI paradigms (Glaser and Düngelhoff, 1984; Starreveld and La Heij, 1996). The rationale of the present investigation relied on the use of the PRP paradigm to generate an analogous asynchrony in the processing subtended in Task 1 and in Task 2 that perhaps is not also functionally analogous to staggering pictures and words under single-task condition. Future research will certainly be of help in addressing at disentangling this crucial point. At present, we favor an explanation based on the present and prior observations suggesting a range of conclusions that seem to converge on the notion that, whereas phonological processing seems to engage, at least in part, central mechanisms, semantic processing does not seem to impose the same demands (e.g., Dell'Acqua et al., 2010). Evidence in support of unimpeded (not engaging central mechanisms) semantic processing comes from studies investigating the attentional blink $(\mathrm{AB})$ phenomenon, which some have proposed to ensue from the same, central, limitations causing the PRP effect (e.g., Jolicoeur and Dell'Acqua, 1998). In the AB paradigm, subjects have to monitor rapid serial visual streams of stimuli for detection of two pre-specified targets, usually labeled as T1 and T2. The critical manipulation in $\mathrm{AB}$ studies is the SOA between $\mathrm{T} 1$ and $\mathrm{T} 2$, which is systematically manipulated by varying the number of distractors intervening between $\mathrm{T} 1$ and $\mathrm{T} 2$. As a result, $\mathrm{T} 1$ is reported in most cases, whereas T2 is missed on most trials when the SOA between T1 and T2 is around 250/300 ms. Studies tracking the ERP response to T2 have shown a striking similar- ity between ERP deflections observed following T2 onset and ERP deflections following the onset of the second stimulus in PRP paradigms. In both cases, the P3b component of such ERP responses is postponed at short vs. long SOA, establishing an important conceptual bridge between $\mathrm{AB}$ and PRP phenomena (e.g., Luck and Vogel, 2001; Dell'Acqua et al., 2005; Sessa et al., 2007). What is worthy of mention and in line with our present interpretative suggestions is that, though missed during the $A B$ because its processing is subject to central processing postponement, T2 is still capable of generating a N400 component, an hallmark of semantic processing (Vogel et al., 1998; Pesciarelli et al., 2007). Though slightly indirect, this findings mesh well with our proposed, pre-central, origin of semantic effects in PWI tasks, and of the dissociation of semantic and central or post-central phonological effects as proposed in prior studies using the PRP paradigm (e.g., Ferreira and Pashler, 2002).

Under the assumption that this pre-central stage of processing can be apprehended like a pre-lexical stage of processing in the PWI, a tentative connection between the attentional mechanisms driving the semantic interference effect and its locus in a broader lexical access context can be proposed. The early pre-central locus of semantic interference does not easily fit within models in which the effect emerges at the lexical competition level (Schriefers et al., 1990; Roelofs, 1992; Levelt et al., 1999), since this stage have been identified as requiring central attentional resources when it was indexed by lexical frequency manipulations (Ferreira and Pashler, 2002; Dent et al., 2008). The response exclusion hypothesis (Mahon et al., 2007) does not seem to provide a better account for the early locus either. Actually, replaced in the context of the PRP paradigm, this hypothesis would seem to locate the semantic interference effect at a post-central stage of processing. Thus, the genuine early locus of the semantic interference effect revealed by our findings seems more in line with hypotheses locating it at pre-lexical processes of message elaboration, rather than at a lexical or even a post-lexical stage (e.g., Costa et al., 2005; Kuipers et al., 2006).

\section{CONCLUSION}

In two experiments, we assessed the classic phonological facilitation and semantic interference effects in a PRP paradigm. We observed that the magnitude of the phonological facilitation effect remained constant across SOAs, while the semantic interference effect were virtually absent at short SOA. These findings allow both empirical and theoretical conclusions. On the empirical side, the reported phonological additive effects, together with the post hoc analyses and other arguments coming from the literature, support the relevance of inserting the PWI paradigm in a PRP design to assess attentional requirements of linguistic processing. On the theoretical side, the findings indicate that the under additive semantic interference effect originally reported by Dell'Acqua et al. (2007a) and replicated here is located at a pre-central locus in a PRP conceptualization of the picture-word task. Given the centrality of the semantic interference effect in current thinking about lexical processing, this conclusion imposes an important constraint for models of lexical access in language production. 


\section{REFERENCES}

Ayora, P., Janssen, N., Dell'Acqua, R., and Alario, F.-X. (2009). Attentional requirements for the selection of words from different grammatical categories. J. Exp. Psychol. Learn. Mem. Cogn. 35, 1344-1351.

Cohen, A., and Magen, H. (2004). "Hierarchies of attention and action," in Attention in Action, eds G. Humphreys and J. Riddoch (Hove: Psychology Press), 27-67.

Cook, A. E., and Meyer, A. S. (2008). Capacity demands of phoneme selection in word production: new evidence from dual-task experiments. J. Exp. Psychol. Learn. Mem. Cogn. 34,886-899.

Costa, A., Alario, F.-X., and Caramazza, A. (2005). On the categorical nature of the semantic interference effect in the picture-word interference paradigm. Psychon. Bull. Rev. 12, 125-131.

Dell'Acqua, R., Job, R., Peressotti, F., and Pascali, A. (2007a). The picture-word interference effect is not a Stroop effect. Psychon. Bull. Rev. 14, 717-722.

Dell'Acqua, R., Pesciarelli, F., Jolicoeur, P., Eimer, M., and Peressotti, F. (2007b). The interdependence of spatial attention and lexical access as revealed by early asymmetries in occipito-parietal ERP activity. Psychophysiology 44, 436-443.

Dell'Acqua, R., and Jolicoeur, P. (2000). Visual encoding of patterns is subject to dual-task interference. Mem. Cognit. 28, 184-191.

Dell'Acqua, R., Jolicoeur, P., Vespignani, F., and Toffanin, P. (2005). Central processing overlap modulates P3 latency. Exp. Brain Res. 165, 54-68.

Dell'Acqua, R., Sessa, P., Peressotti, F., Mulatti, C., Navarrete, E., and Grainger, J. (2010). ERP evidence for ultra-fast semantic processing in the picture-word interference paradigm. Front. Psychol. 1:177. doi: 10.3389/ fpsyg.2010.00177

Dent, K., Johnston, R.A., and Humphreys, G. W. (2008). Age of acquisition and word frequency effects in picture naming: a dual-task investigation. $J$. Exp. Psychol. Learn. Mem. Cogn. 34, 282-301.
Ferreira, V. S., and Pashler, H. (2002). Central bottleneck influences on the processing stages of word production. J. Exp. Psychol. Learn. Mem. Cogn. 28, 1187-1199.

Glaser, W. R., and Düngelhoff, F. J. (1984). The time course of picture-word interference. J. Exp. Psychol. Hum. Percept. Perform. 10, 640-654.

Jolicoeur, P., and Dell'Acqua, R. (1998) The demonstration of short-term consolidation. Cogn. Psychol. 36, 138-202.

Kuipers, J.-R., La Heij, W., and Costa, A. (2006). A further look at semantic contexts effects in language production: the role of response congruency. Lang. Cogn. Process. 21, 892-919.

Laudanna, A., Thornton, A. M., Brown, G., Burani, C., and Marconi, L. (1995). "Un corpus dell'italiano scritto contemporaneo dalla parte del ricevente," in Giornate Internazionali di Analisi Statistica dei Dati Testuali, Vol. I, eds S. Bolasco, L. Lebart, and A. Salem III (Roma: Cisu), 103-109.

Levelt, W. J. M., Roelofs, A., and Meyer, A. S. (1999). A theory of lexical access in speech production. Behav. Brain Sci. $22,1-75$.

Lien, M.-C., Cornett, L., Goodin, Z., Ruthruff, E., and Allen, P. A. (2008). On the nonautomaticity of visual word processing: electrophysiological evidence that word processing requires central attention. J. Exp. Psychol. Hum. Percept. Perform. 34, 751-773.

Luck, S. J., and Vogel, E. K. (2001). "Multiple sources of interference in dual-task performance: the case of the attentional blink and the psychological refractory period," in The Limits of Attention, ed. K. L. Shapiro (New York: Oxford University Press), 124-140.

Mahon, B. Z., Costa, A., Peterson, R. Vargas, K. A., and Caramazza, A. (2007). Lexical selection is not by competition: a reinterpretation of semantic interference and facilitation effects in the picture-word interference paradigm. J. Exp. Psychol. Learn. Mem. Cogn. 33, 503-535.

Meyer, A. S., and Schriefers, H. (1991). Phonological facilitation in pictureword interference experiments: effects of stimulus onset asynchrony and types of interfering stimuli. $J$. Exp. Psychol. Learn. Mem. Cogn. 17 1146- 1160

Meyer, D. E., and Kieras, D. E. (1997). A computational theory of executive $\operatorname{cog}$ nitive processes and human multipletask performance: Part 2. Accounts of psychological refractory-period phenomena. Psychol. Rev. 104, 749-791.

Miozzo, M., and Caramazza, A. (2003). When more is less: a counterintuitive effect of distractor frequency in the picture-word interference paradigm. J. Exp. Psychol. Gen. 132, 228-258.

Pashler, H. (1994). Dual-task interference in simple tasks: data and theory. Psychol. Bull. 116, 220-244.

Pashler, H., and Johnston, J. C. (1989). Chronometric evidence for central postponement in temporally overlapping tasks. Q. J. Exp. Psychol. 41A, 19-45.

Pashler, H., and Johnston, J. C. (1998) "Attentional limitations in dual-task performance," in Attention, ed. H. Pashler (Hove: Psychology Press), 155-189.

Pesciarelli, F., Kutas, M., Dell'Acqua, R. Peressotti, F., Job, R., and Urbach, T. P. (2007). Semantic and repetition priming within the attentional blink an event-related brain potential (ERP) investigation. Biol. Psychol. 76, 21-30.

Reiner, M. B., and Morrison, F. J. (1983) Is semantic interference really automatic? Bull. Psychon. Soc. 21, 271-274.

Roelofs, A. (1992). A spreading-activation theory of lemma retrieval in speaking. Cognition 42, 107-142.

Roelofs, A. (2008). Attention, gaze shifting, and dual-task interference from phonological encoding in spoken word planning. J. Exp. Psychol. Hum. Percept. Perform. 34, 1580-1598.

Ruthruff, E., Pashler, H. E., and Klaassen, A. (2001). Processing bottlenecks in dual-task performance: structural limitation or voluntary postponement? Psychon. Bull. Rev. 8, 73-80.

Schneider, W., Eschman, A., and Zuccolotto, A. (2002). E-Prime User's Guide. Pittsburgh: Psychology Software Tools, Inc.
Schriefers, H., Meyer, A. S., and Levelt, W. J.M. (1990). Exploring the time course of lexical access in language production: picture-word interference studies. J. Mem. Lang. 29, 86-102.

Sessa, P., Luria, R., Verleger, R., and Dell'Acqua, R. (2007). P3 latency shifts in the attentional blink: further evidence for second target processing postponement. Brain Res. 1137, 131-139.

Starreveld, P. A., and La Heij, W. (1996). Time-course analysis of semantic and orthographic context effects in picture-naming.J. Exp. Psychol. Learn. Mem. Cogn. 22, 896-918.

Van Selst, M., and Jolicoeur,P. (1994).Can mental rotation occur before the dualtask bottleneck? J. Exp. Psychol. Hum. Percept. Perform. 20, 905-921.

Vogel, E. K., Luck, S. J., and Shapiro, K. L. (1998). Electrophysiological evidence for a postperceptual locus of suppression during the attentional blink. J. Exp. Psychol. Hum. Percept. Perform. 24, 1656-1674.

Conflict of Interest Statement: The authors declare that the research was conducted in the absence of any commercial or financial relationships that could be construed as a potential conflict of interest.

Received: 14 December 2010; accepted: 24 March 2011; published online: 06 April 2011.

Citation: Ayora P, Peressotti F, Alario $F-X$, Mulatti C, Pluchino P, Job R and Dell'Acqua R (2011) What phonological facilitation tells about semantic interference: a dual-task study. Front. Psychology 2:57. doi: 10.3389/fpsyg.2011.00057 This article was submitted to Frontiers in Language Sciences, a specialty of Frontiers in Psychology.

Copyright $\odot 2011$ Ayora, Peressotti, Alario, Mulatti, Pluchino, Job and Dell'Acqua. This is an open-access article subject to a nonexclusive license between the authors and Frontiers MediaSA, which permits use, distribution and reproduction in other forums, provided the original authors and source are credited and other Frontiers conditions are complied with. 


\section{APPENDIX EXPERIMENT 1}

\begin{tabular}{|c|c|c|c|}
\hline Pictures & Begin-related & End-related & Unrelated \\
\hline BANANA (banana) & Banconota (bill) & Settimana (week) & Ambulatorio (surgery) \\
\hline BICCHIERE (glass) & Bidello (janitor) & Infermiere (nurse) & Suola (sole) \\
\hline BICICLETTA (bike) & Bistecca (steak) & Disdetta (cancelation) & Dinamite (dynamite) \\
\hline BOTTIGLIA (bottle) & Bottega (shop) & Meraviglia (astonishment) & Sbaglio (mistake) \\
\hline CAMMELLO (camel) & Camino (cimney) & Gioiello (jewel) & Scultura (sculpture) \\
\hline CANGURO (kangaroo) & Canale (canal) & Muro (wall) & Mobile (furniture) \\
\hline CAPPELLO (hat) & Capanna (hut) & Pennello (paintbrush) & Raffica (gust) \\
\hline CARCIOFO (artichoke) & Cappa (voult) & Filosofo (philosopher) & Lanterna (lantern) \\
\hline CAROTA (carrot) & Cartolina (postcard) & Pilota (pilot) & Delizioso (delicious) \\
\hline ELEFANTE (elephant) & Elettore (voter) & Contante (cash) & Telo (piece of cloth) \\
\hline ELICOTTERO (helicopter) & Elenco (list) & Zucchero (sugar) & Agenzia (agency) \\
\hline FRAGOLA (strawberry) & Frangia (fringe) & Nuvola (cloud) & Civetta (owl) \\
\hline FRECCIA (arrow) & Fratello (brother) & Goccia (drop) & Istituto (institute) \\
\hline GALLINA (hen) & Garage (garage) & Benzina (petrol) & Cornice (frame) \\
\hline GONNA (skirt) & Goloso (greedy) & Pinna (fin) & Palato (palate) \\
\hline GUANTO (glove) & Guadagno (income) & Schianto (crash) & Elefante (elephant) \\
\hline IMBUTO (funnel) & Impronta (imprint) & Velluto (velvet) & Battesimo (baptism) \\
\hline LEONE (lion) & Letargo (hibernation) & Sapone (soap) & Carovana (caravan) \\
\hline LETTO (bed) & Leggenda (legend) & Ghetto (ghetto) & Ombrello (umbrella) \\
\hline PERA (pear) & Percorso (path) & Miniera (mine) & Concorso (competition) \\
\hline PINGUINO (penguin) & Pizza (pizza) & Contadino (farmer) & Trasloco (move) \\
\hline PISTOLA (gun) & Piramide (pyramid) & Suola (sole) & Argine (bank) \\
\hline SCIARPA (scarf) & Scimmia (monkey) & Arpa (harp) & Pacco (pack) \\
\hline SCOIATTOLO (squirrel) & Scodella (bowl) & Prezzemolo (parsley) & Lancette (clock hands) \\
\hline SEDIA (chair) & Seme (seed) & Invidia (envy) & Lenzuola (sheet) \\
\hline STIVALI (boots) & Stemma (coat of arms) & Cereali (cereals) & Raso (satin) \\
\hline STRUZZO (ostrich) & Stretto (narrow) & Attrezzo (tool) & Disco (disk) \\
\hline TAVOLO (table) & Talento (talent) & Idolo (idol) & Fresco (fresh) \\
\hline TOPO (mouse) & Torta (cake) & Scopo (aim) & Ginnastica (gymnastics) \\
\hline TRATTORE (tractor) & Tregua (truce) & Splendore (splendor) & Pomodoro (tomato) \\
\hline TRENO (train) & Trespolo (trestle) & Fieno (hay) & Scamorza (scamorza cheese) \\
\hline TRICICLO (tricycle) & Tribuna (platform) & Riciclo (recycling) & Vasca (tank) \\
\hline TROMBA (trumpet) & Trucco (make-up) & Gamba (leg) & Soldato (soldier) \\
\hline ZEBRA (zebra) & Zeppa (wedge) & Fibra (fiber) & Tasto (key) \\
\hline
\end{tabular}




\section{EXPERIMENT 2}

\begin{tabular}{|c|c|c|c|}
\hline Pictures & Phonologically related & Semantically related & Unrelated \\
\hline BANANA (banana) & Bancone (counter) & Arancia (orange) ${ }^{\mathrm{a}}$ & Maglione (sweather) ${ }^{c}$ \\
\hline BICCHIERE (glass) & Bidello (janitor) & Coltello (knife) ${ }^{\mathrm{a}}$ & Grattacielo (skyscraper) ${ }^{c}$ \\
\hline BICICLETTA (bike) & Bistecca (steak) & Camper (camper van) & Cassetto (drawer) ${ }^{c}$ \\
\hline CANGURO (kangaroo) & Candela (canddle) & Giraffa (giraffe) $^{b}$ & Pentola (pot) ${ }^{c}$ \\
\hline CAPPELLO (hat) & Capanna (hut) & Pantaloni (trousers) ${ }^{\mathrm{a}}$ & Albicocca (apricot) ${ }^{c}$ \\
\hline CARCIOFO (artichoke) & Carbone (coal) & Piselli (peas) ${ }^{\mathrm{a}}$ & Bastone $(\text { stick })^{c}$ \\
\hline CAROTA (carrot) & Carrello (trolley) & Sedano (celery) & Tenda (tent) ${ }^{c}$ \\
\hline CHIESA (church) & Chicco (grain) & Torre (tower) ${ }^{\mathrm{a}}$ & Palma $\left(\right.$ palm) ${ }^{c}$ \\
\hline GALLINA (hen) & Garage (garage) & Anatra (duck) & Forbice (scissors) ${ }^{c}$ \\
\hline GONNA (skirt) & Goloso (greedy) & Canottiera (singlet) ${ }^{a}$ & Zaino (knapsack) c \\
\hline GUANTO (glove) & Guadagno (income) & Cintura (belt) ${ }^{\mathrm{a}}$ & Birillo (skittle) ${ }^{c}$ \\
\hline IMBUTO (funnel) & Impronta (imprint) & Mestolo (ladle) ${ }^{\mathrm{a}}$ & Camino $\left(\right.$ cimney) ${ }^{\circ}$ \\
\hline LIMONE (lemon) & Limite (limit) & Prugna (plum) & Scarpa (shoe) ${ }^{c}$ \\
\hline MAIALE (pig) & Malaria (malaria) & Pecora (sheep) & Cipolla (onion) \\
\hline NOCE (walnut) & Notaio (notary) & Castagna (chestnut) ${ }^{\mathrm{a}}$ & Spada $\left(\right.$ sword) ${ }^{c}$ \\
\hline PEPERONE (pepper) & Persiana (shutter) & Fungo (mushroom) & Ombrello (umbrella) ${ }^{c}$ \\
\hline PERA (pear) & Pelo (hair) & Fico $(f i g)^{b}$ & Mucca $(\operatorname{cow})^{c}$ \\
\hline PINGUINO (penguin) & Pineta (pinewood) & Aquila (eagle) ${ }^{a}$ & Cancello (gate) $^{c}$ \\
\hline TROMBA (trumpet) & Tronco (trunk) & Pianoforte (piano) & Foglia (leaf) ${ }^{c}$ \\
\hline ZEBRA (zebra) & Zeppa (wedge) & Gatto (cat) & Tasto (key) \\
\hline
\end{tabular}

Letters indicate which words were previously used in Dell'Acqua et al. (2007a):

a Semantically related distractor words associated to the same picture.

${ }^{b}$ Semantically related distractors words associated to a different picture.

c Unrelated distractor words associated to a different picture. 